\title{
Neumonía adquirida en la comunidad
}

Lado Lado FL. Neumonía adquirida en la comunidad. An Med Interna (Madrid) 2002; 19: 609-611.

La neumonía adquirida en la comunidad (NAC) se define como la infección del parénquima pulmonar que, originada en el ámbito extrahospitalario, cursa con fiebre, clínica respiratoria variable e infiltrados radiológicos pulmonares. Esta patología constituye una causa muy frecuente de consulta médica, no exenta de una importante morbimortalidad. Habitualmente, el tratamiento inicial de la NAC se realiza de forma empírica. Para ello se ha de tener en cuenta, por una parte, la epidemiología y el patrón de resistencias de la propia comunidad y, por otra, la presencia de diferentes aspectos como la edad, comorbilidad, inmunosupresión y/o la severidad del cuadro clínico.

En el presente número de Anales de Medicina Interna se publican sendos artículos de esta materia. En uno de ellos, Nuñez Fernández y cols. (1) aportan un estudio retrospectivo sobre la neumonía neumocócia con bacteriemia, matizando los factores predisponentes de la enfermedad: un incremento de la enfermedad ante la presencia de la infección por el virus de la inmunodeficiencia humana (VIH) en la población con edad inferior a los 40 años, una resistencia del neumococo del $31 \%$ y una mortalidad del $10 \%$ relacionada, especialmente, con un índice de severidad superior a 140. En el otro, Geijo Martínez y cols. (2) elaboran un estudio prospectivo sobre el empleo de Levofloxacino en monoterapia, frente al tratamiento combinado con betalactámico y macrólido en grupos de pacientes de similares caracteríticas Al tiempo, se recalca un elevado porcentaje de bacilos gramnegativos, una gravedad inicial prominente con mortalidad del $5 \%$ y una mayor necesidad de prolongar el tratamiento en el grupo stándar.

De hecho, la NAC no es una enfermedad de declaración obligatoria, por lo que el porcentaje real de casos se desconoce. No obstante, en función de las comunidades analizadas, se estima que su incidencia anual puede oscilar en torno a 1,6 y 12 casos por 1.000 habitantes, con mayor relevancia en los grupos de edad comprendidos en ambos extremos de la vida (3-5). En EE.UU., la NAC representa la sexta causa de mortalidad y el primer motivo de fallecimiento por enfermedad infecciosa. Se considera que la tasa de mortalidad, en los pacientes hospitalizados, varía entre el 10 y el $25 \%$ y, estos valores aumentan considerablemente en los casos que requieren ingreso en UCI $(6,7)$.
En cuanto a su origen, no siempre se identifica el agente causal, que en la mayoría de los casos suele ser único y a veces puede ser producido por 2 o más microorganismos. En la etiología, el Streptococcus pneumoniae representa el patógeno más usual, seguido de un amplio espectro de microorganismos atípicos tales como la Chlamydia pneumoniae, el Myco plasma pneumoniae, la Legionella pneumophila y la Coxiella Burnetii. Otros agentes que debemos citar son la Moraxella catharralis, el Haemophilus influenzae, el Staphylococccus aureus y la Klebsiella pneumoniae. También se han visto implicados los virus respiratorios. Sin embargo, el espectro fluctúa dependiendo de la edad, época estacional y de la zona estudiada, y así, por ejemplo, en nuestro país, la L. pneumop hila es muy común en el área mediterránea. En las neumonías graves que requieren ingreso en UCI, los patógenos responsables más reiterados son el $S$. pneumoniae, la L. pneumophila y los bacilos gramnegativos (4,8-10).

La NAC, aunque está descrita en todos los grupos de edad y puede aparecer en personas previamente sanas, afecta, fundamentalmente, a las de edad avanzada, a las que poseen hábitos tóxicos como el tabaquismo y el alcoholismo, y a las que padecen enfermedades crónicas o debilitantes. En este último grupo, incluímos la enfermedad pulmonar obstructiva crónica, la diabetes mellitus, la insuficiencia renal crónica, las neoplasias, la insuficiencia cardiaca congestiva, la cardiopatía isquémica, las enfermedades hepáticas crónicas o las enfermedades neurológicas. Además, la NAC tiene predilección por los enfermos con inmunodeficiencia, como los infectados por el VIH o los que están sometidos a un tratamiento inmunosupresor $(9,11,12)$.

Estas situaciones especiales contribuyen, en gran medida, a la orientación diagnóstica, pronóstica y terapéutica de la NAC porque pueden favorecer la agresividad de los agentes etiológicos responsables y, en consecuencia, se intensifican tanto las complicaciones como las cifras de mortalidad. Este hecho, resulta evidente en los casos de neumonía neumocócica con bacteriemia en los que, comparativamente con los que no tienen bacteriemia, se observan: mayores factores predisponentes, en particular el alcoholismo, una extensión radiológica más amplia y un desarrollo de las manifestaciones extrapulmonares (12). 
Para el diagnóstico de la NAC, se requiere el estudio microbiológico de muestras adquiridas, normalmente, por métodos incruentos. Dentro de ellos, las tomas de esputo son procesadas para tinción de Gram y cultivo que, entre un $10 \%$ y un $30 \%$ de las ocasiones, son difíciles de obtener por ausencia de expectoración. Los hemocultivos, que deben practicarse en la mayoría de los ingresos en el hospital, llegan a alcanzar una rentabilidad del $67 \%$ si el bacilo responsable es el S. pneumoniae. Asimismo, podemos emplear otros métodos como la realización de tests serológicos y de aglutinación. En determinadas situaciones relevantes, con curso clínico complicado, que requieren un diagnóstico definitivo, resultan eficaces ciertas pruebas entre las que destacan la detección de antígenos o la PCR. No obstante, en algunas circunstancias, los mencionados análisis no nos permiten llegar a un diagnóstico concluyente, y nos podemos ver en la obligación de conseguir muestras por métodos invasivos. En este apartado, citamos la broncoscopia, la aspiración transtraqueal y la punción percutánea pulmonar aspirativa; de este modo, el procesamiento de las muestras logradas serían eficientes para orientar el tratamiento inicial $(7,12,13)$.

Para establecer la severidad de la NAC nos basamos, primordialmente, en dos pilares que son las pruebas de laboratorio y la valoración clínica inicial. El primero de ellos, contiene la realización de gasometría arterial, hemograma, bioquímica y radiología de tórax, que nos informan sobre la existencia de acidosis, hipoxemia, retención carbónica, grado de leucocitosis o leucopenia, la tasa de urea, la extensión radiológica, afectación de uno o más lóbulos con otras alteraciones asociadas como derrame pleural. En el segundo, nos guiamos por dos principios fundamentales como la frecuencia respiratoria y la presión arterial, que pueden ser medidos en el momento del diagnóstico y en cualquier situación, siendo, ambos, de gran utilidad pronóstica. A modo de ejemplo, una frecuencia de $\geq 30$ respiraciones por minuto y una presión arterial diastólica de $60 \mathrm{mmHg}$ son datos indicativos de severidad del cuadro clínico. Otros elementos pronósticos implicados son el estado nutricional, el estado mental y el tratamiento antibiótico aplicado. Más concretamente, en el grupo de NAC graves, que requieren ingreso en UCI se han descrito, tambien, el shock séptico, la extensión radiológica con afectación multilobular y la necesidad de ventilación mecánica $(7,14-16)$.

Durante los últimos años, el tratamiento empírico de la NAC ha ido evolucionando gradualmente. Este hecho se suscita tanto por la aparición de resistencias antibióticas hacia algunos de los gérmenes habituales, cuanto por la aparición de nuevos agentes etiológicos. Por este motivo, se han diseñado diferentes pautas de tratamiento antibiótico empírico que, ordinariamente, engloban la prescripción de una cobertura antibiótica de amplio espectro en función de la edad, características epidemiológicas, la situación clínica, comorbilidad y el estado de resistencias.

Por lo que se refiere al patógeno etiológico más habitual de la NAC que es el $S$. pneumoniae, en las últimas décadas, comenzó a desarrollar un incremento de las resistencias frente a múltiples agentes antimicrobianos: betalactámicos, macrólidos, cloramfenicol, tetraciclinas, trimetropin-sulfametoxazol y aminoglucósidos. Dentro de aquellas, la más importante es la resistencia a la penicilina y a otros betalactámicos, dada la función que desempeñan en el tratamiento de la infección neumocócica. Asimismo, interesa la resistencia a los macrólidos, porque estos antimicrobianos habían sustituido a los betalactámicos en el tratamiento de la infección $(10,17,18)$.

En la actualidad, se han añadido, al conjunto terapeútico ya existente, las nuevas quinolonas de tercera y de cuarta genera- ción. Estos fármacos presentan un amplio espectro y tienen numerosas ventajas, sobre todo desde el punto de vista farmacocinético. En esta línea, se caracterizan por una vida media prolongada, que permite una única dosis diaria, buena tolerancia y escasos efectos secundarios a excepción de hepatotoxicidad relacionada con trovafloxacino. La vía de administración puede ser bien oral bien intravenosa; ello facilita efectuar la terapia de modo secuencial y, en consecuencia, acortar la estancia hospitalaria. En realidad, los primeros estudios publicados sobre la utilización de levofloxacino en la NAC demuestran una efectividad ligeramente superior a las pautas clásicas aportando, además, la ventaja de un aminoramiento de los costes $(4,8,19-23)$.

La quinolonas de tercera y cuarta generación cubren un amplio espectro de microorganismos, y no sólo poseen una mayor actividad frente a cocos grampositivos (incluidas las cepas de neumococo resistente a betalactámicos), sino que mantienen la eficacia de las quinolonas antiguas contra el resto de los patógenos respiratorios. Por tanto, las quinolonas de tercera generación, como levofloxacino, abarcan la práctica totalidad de las bacterias causantes de la NAC, esto es el $S$. pneumoniae y diversos grampositivos, los microorganismos atípicos (Mycoplasma, Chlamydia, Coxiella, Legionella), enterobacterias y la $P$. Aeruginosa. En cuanto a las quinolonas de cuarta generación, como Moxifloxacino, también son potentes frente a los gérmenes anaerobios $(4,8,20)$.

Por lo que se refiere a la indicación del uso de quinolonas en las dispares pautas de recomendaciones y guías terapéuticas, existen ciertas discrepancias, probablemente, por las diferencias habidas en la distribución de los agentes etiológicos y las resistencias entre las poblaciones analizadas (24). En atención a la severidad de la NAC, se puede optar por la monoterapia o la terapia combinada con otros agentes antimicrobianos en los grupos de mayor riesgo. En las neumonías leves, la Infectious Diseases Society of America (IDSA) incluye, a las quinolonas, como opción a macrólido o doxiciclina, indicándolas de elección en pacientes con comorbilidad o de edad avanzada; Canadian Infec tious Disease Society/Canadian Thoracic Society (CIDS/CTS) recomiendan su uso de primera elección, en circunstancias tales como antecedentes recientes de tratamientos con otros agentes antimicrobianos o con corticoides; y la American Thoracic Society (ATS) las apuntan como alternativa a otras pautas, en determinadas situaciones como enfermedad cardiopulmonar, y a expensas de las tasa de resistencias locales al neumococo (2527). Sin embargo, hay que recalcar que ya se han descrito resistencias durante el tratamiento con levofloxacino (28).

A modo de conclusión, podríamos afirmar que, pese a los avances acontecidos en los últimos años en cuanto a la disponibilidad de modernos métodos diagnósticos y de nuevos agentes antimicrobianos, la NAC continúa teniendo una importancia extraordinaria en lo relativo a la morbimortalidad que se produce, de manera más notable, en pacientes portadores de otras patologías o los de edad avanzada. Por ello, se debe ahondar en un profundo conocimiento de los factores epidemiológicos, las tasas de resistencias y una aplicación racional de los medios disponibles a nuestro alcance.

\section{F. L. LADO LADO}

Servicio de Medicina Interna. Hospital Clínico Universitario. Departamento de Medicina. Universidad de Santiago de Compostela. Santiago de Compostela. A Coruña. 


\section{Bibliografía}

1. Núñez Fernández MJ, Ojea de Castro R, Lueiro Lores F, Pulian Morais MV, Hernández Blanco M, Núñez Fernández M, De Lis Muñoz JM. Neumonía neumocócica con bacteriemia en adultos: estudio descriptivo en el noroeste de España. An Med Interna (Madrid) 2002; 19: 612-20.

2. Geijo Martínez MP, Meiling Díaz de Tuesta A, Herranz CR, Gómez Criado CG, Dimas Núñez JF, Saiz García F. Levofloxacino frente a betalactámicos en el tratamiento de la neumonía adquirida en la comunidad con ingreso hospitalario. An Med Interna ( Madrid) 2002; 19: 621-5.

3. Jokinen C, Heiskanen L, Junoven H, Kallinen S, Karkola K, Korppi M, et al. Incidence of community-acquired pneumonia in the population of four municipalities in Eastern Finland. Am J Epidemiol 1993; 137: 977 88 .

4. Guthrie R. Community-acquired lower respiratory tract infections. Etiology and treatment. Chest 2001; 120: 2021-34.

5. Almirall J, Bolilbar I, Vidal J, Sauca G, Coll P, Niklasson B, et al. Epidemiology of community-acquired pneumonia in adults: a populationbased study. Eur Respir J 2000; 15: 757-63.

6. Garibaldi RA. Epidemiology of community-acquired respiratory tract infections in adults: incidence, etiology, and impact. Am J Med 1985; 78: 32S-7S

7. Bartlett JG, Mundy LM. Community-acquired pneumonia. N Engl J Med 1995; 333: 1618-24.

8. Dorca J, Zalacain R. Avances en el tratamiento antimicrobiano de los pacientes con neumonía adquirida en la comunidad. Med Clin (Barc) 2001; 2 (Supl 2): 29-34.

9. Lim WS, Macfarlane JT, Boswell TCJ, Harrison TG, Rose D, Leinonen M, Saikku P. Study of community acquired pneumonia aetiology (SCAPA) in adults admitted to hospital: implications for management guidelines. Thorax 2001; 56: 296-301.

10. Woodhead W. Community-acquired pneumonia in Europe: causative pathogens and resistance patterns. Eur Respir J 2002; 20 (Suppl 36): 20S-27S.

11. Magnenat JL, Nicod LP, Auckenthaler R, Junod AF. Mode of presentation and diagnosis of bacterial pneumonia in human immunodeficiency virus-infected patients. Am Rev Respir Dis 1991; 144: 917-22.

12. Musher DM, Alexandraki I, Graviss EA, Yanbeiy N, Eid A, Inderias LA, et al. Bacteremic and nonbacteremic pneumococcal pneumonia. A prospective study. Medicine 2000; 79: 210-21.

13. Ayerbe R, Verano A, Capote F, Pachón J, Cuello JA, Segado A. Punción aspirado pulmonar en el diagnóstico etiológico de la neumonías. Rev Clin Esp 1994; 194: 270-5.

14. Marrie TJ, Durant H, Yates L. Community-acquired pneumonia requiring hospitalization: 5 year prospective study. Rev Infect Dis 1989; 11: 586-99.
15. Fine MJ, Auble ThE, Yealy DM, Hanusa BH, Weissfeld LA, Singer DE, et al. A prediction rule to identify low-risk patients with community-acquired pneumonia. N Engl J Med 1997; 336: 243-50.

16. Ewig S, Ruiz M, Mensa J, Marcos MA, Martínez JA, Arancibia F, Niederman MS, Torres A. Severe community-acquired pneumonia: assessment of severity criteria. Am J Respir Crit Care 1998; 158: 1102-8.

17. Harwell JI, Brown RB. The drug-resistant pneumococcus. Clinical relevance, therapy, and prevention. Chest. 2000; 117: 530-41.

18. Klugman KP. Bacteriological evidence of antibiotic faliure in pneumonoccal lower respiratory tract infections. Eur Respir J 2002; 20 (Suppl 36): $3 \mathrm{~S}-8 \mathrm{~S}$.

19. Arancibia F, Maldonado A, Torres A. Experiencia clínica de levofloxacino en la neumonía adquirida en la comunidad. Enferm Infecc Microbiol Clin 1999; 17 (Supl 1): 14-18.

20. File TM. Levofloxacin in the treatment of community acquired pneumonia. Can Respir J. 1999; 6 (Suppl A): 35A-9A.

21. Clark LC, Davis CW. Experiences at a large teaching hospital with levofloxacin for the treatment of community-acquired pneumonia. Am J Health-Syst Pharm 2000; 57 (Suppl 3): 10S-13S.

22. Torres A. Nuevas quinolonas en el tratamiento de la neumonía comunitaria no hospitalizada. Rev Esp Quimioterap 2000; 13 (Supl 1): 19-27.

23. Marrie TJ, Lau CY, Wheeler SL, Wong CJ, Vandervoort MK, Feagan BG. A controlled trial of a critical pathway for treatment of communityacquired pneumonia. CAPITAL Study Investigators. CommunityAdquired Pneumonia Intervention Trial Assessing Levofloxacin. JAMA 2000; 283: 749-55.

24. Mandell LA. Guidelines fos community-adquired pneumonia: a tale of 2 countries. Clin Infect Dis 2000; 31: 422-5.

25. Bartlett JG, Dowell SF, Mandell LA, File TM, Musher DM, Fine MJ. Practice guidelines for the management of community-acquired pneumonia. Clin Infect Dis 2000; 31: 347-82.

26. Mandell LA, Marrie ThJ, Grossman RF, Chow AW, Hyland RH and the Canadian community-acquired pneumonia working group. Canadian guidelines for the initial management of community-acquired pneumonia: an evidence-based update by the Canadian infectious diseases society and the Canadian thoracic society. Clin Infect Dis 2000; 31: $383-421$

27. American Thoracic Society. Guidelines for the management of adults with community-acquired pneumonia. Am J Respir Crit Care Med 2001; 163: 1730-54

28. Davidson R, Cavalcanti R, Brunton JL, Bast DJ, De Azevedo JCS, Kibsey $\mathrm{P}$, et al. Resistance to levofloxacin and failure of treatment of pneumococcal pneumonia. N Engl J Med 2002; 346: 747-50. 